\title{
Análisis del estado poblacional de los arracachales Montrichardia arborescens (L) Schott (Araceae) en la llanura aluvial de la cuenca del Atrato, Chocó, Colombia
}

\section{Analysis of the population condition of the arracachales of Montrichardia arborescens (L) Schott (Araceae), in the alluvial plain of the Atrato basin, Chocó, Colombia}

\section{Zulmary Valoyes Cardozo', Giovanny Ramírez Moreno² \\ Resumen}

Se estudió una población de Montrichardia arborescens en tres localidades (Carmen de Darién, Riosucio y Unguía), correspondientes a un bosque inundable de la llanura aluvial del río Atrato. En cada localidad se delimitaron cinco parcelas temporales de $20 \times 50 \mathrm{~m}$ cada una, bajo un muestreo aleatorio simple al azar. Se determinó la estructura y la densidad de la especie por localidad y estadio de desarrollo. Los resultados de este estudio registran valores de hasta 7.367 individuos para el estadio plántula y 6.000 individuos para el estadio adulto, evidenciando una población casi homogénea con densidades generales de 5,5 ind $/ \mathrm{m}^{2}$, corroborando asi que aunque en la zona se realizan actividades de tipo extractivas y a pesar de que el arracacho es una especie promisoria, aún es subvalorada y sus poblaciones se mantienen estables.

Palabras clave: Arracachales, Especie promisoria, Llanura aluvial del Atrato, Montrichardia arborescens.

\begin{abstract}
A population was studied of Montrichardia arborescens in three localities (Carmen of Darién, Riosucio y Unguia), correspondents to a forest inundable of the alluvial plain of the river Atrato. Five temporary plots were established of $20 \times 50 \mathrm{~m}$ each one, under a random simple sampling at random. There decided the structure and the density of the species for locality and stadium of development and 6000 individuals for the adult stadium, demonstrating an almost homogeneous population with general densities of 5, $5 \mathrm{ind} / \mathrm{m}^{2}$, corroborating so though in the zone extractive activities of type realize and to sorrow that the arracacho is a promissory species still, it is an undervalued species and his population are kept stable.
\end{abstract}

Bióloga, Especialista en Administración de Recursos Naturales, Estudiante de Maestría en Ciencias Ambientales. Investigadora principal componente ecosistémico, Instituto de Investigaciones Ambientales del Pacífico (IIAP), Quibdó, Colombia. e-mail: zvaloyes@iiap.org.co

2 Biólogo, Magíster en Ciencias Biológicas, Subdirector de Investigaciones, Instituto de Investigaciones Ambientales del Pacífico (IIAP), Quibdó, Colombia. e-mail:gramirez@iiap.org.co 
Keywords: Alluvial plain of the Atrato river, Arracachales, Montrichardia arborescens, Promissory species.

\section{Introducción}

Montrichardia arborescens (L) Schott (Araceae), es una macrófita acuática anfibia distribuida en el trópico americano que incluye las islas del Caribe (Belice, Dominica, Grenada (Cook 1996). En Colombia se distribuye ampliamente en la Amazonia y el departamento del Chocó donde se le conoce popularmente como aninga o arracaho; forma densas poblaciones en la llanura aluvial inundable del río Atrato, desde Cupica hasta el sur del Parque Natural Nacional Los Katíos, entre el Golfo de Urabá y la desembocadura del río Murrí (Rangel 2004a).

Es una hierba perenne emergente arborescente (helogeófito) con tallos gruesos, crece en ambientes inundados permanentemente, bordeando ríos, ciénegas y caños, donde forma densas poblaciones casi homogénea, con una altura frecuentemente entre 2 y $3 \mathrm{~m}$, algunas otras entre 7 y $10 \mathrm{~m}$, soportados por raíces adventicias; produce rizomas gruesos de 40 a $105 \mathrm{~cm}$ de largo, con perímetro de 12 a $30 \mathrm{~cm}$ (Gordon et al. 2013). En el río Atrato alcanza densidades de 10 a 15 individuos por $\mathrm{m}^{2}$, lo que tiende a formar masas densas monoespecíficas de arracacho (Aldana 2011).

Una de las características de las áreas dominadas por $M$. arborescens, es la tendencia a formar áreas de carácter monoespecífico, que producen las diferencias en número (densidad), altura y grosor de la caña, entre un área y otra. Esta especie forma asociaciones denominadas herbazales/matorrales de arracachales, los cuales se distribuyen a lo largo del corredor fluvial del río Atrato, asociadas con el sistema de ciénagas donde sus zonas pantanosas aún conservan vegetación natural sin intervención, representadas en comunidades vegetales acuáticas y de pantanos; se encuentra asociada con especies como Blechnum serrulatum, Acrostichum aureum, Scleria secans, Scleria melaleuca y especies de Thelypteris y de Panicum (Prieto et al. 2004, Rangel 2004b).

Ecológicamente se constituye como fuente de energía y materiales para otros niveles tróficos de la comunidad, ya sea mediante el proceso de descomposición, el cual estimula la producción y suministra materiales orgánicos e inorgánicos a la cadena de detritus o a través del consumo directo de diferentes partes de la planta (Peña 2000). El Arracacho es considerado en la región como una especie promisoria con potencial para la producción de pulpa para papel; en otras regiones tiene otro tipos de usos como en la medicina; es tóxico, se utiliza como ornamento y para la reforestación, además de proporcionar sombra, refugio y alimento para la fauna silvestre; se considera una fuente importante para la elaboración de papel (Gordon y Pardo 2012), aunque en la actualidad no se aprovechan los individuos de esta especie; sus poblaciones han sido diezmadas para la apertura de canales hacia las ciénagas y pantanos, para desecar los pantanos como vías para acceder al río para el transporte de maderas.

Los arracachales del bajo Atrato hacen parte de la estructura ecológica principal del Chocó Biogeográfico, debido a las extensas áreas de bosque natural que forman sus poblaciones en la planicie de la parte baja del río Atrato, desempeñan un papel fundamental como hábitat y fuente de alimento para las especies residentes u occasionales (Valoyes et al. 2012). Por lo anterior se formula la siguiente pregunta. ¿Existe una diferencia significativa en la estructura de las poblaciones de $M$. arborescens en el Medio y Bajo Atrato?

\section{Metodología}

Área de estudio. Se localizó en la cuenca baja delAtrato en los municipios del Carmen de Darién, Riosucio y Unguía (Figura 1); estos municipios se encuentran inmersos dentro de una red de sis- 


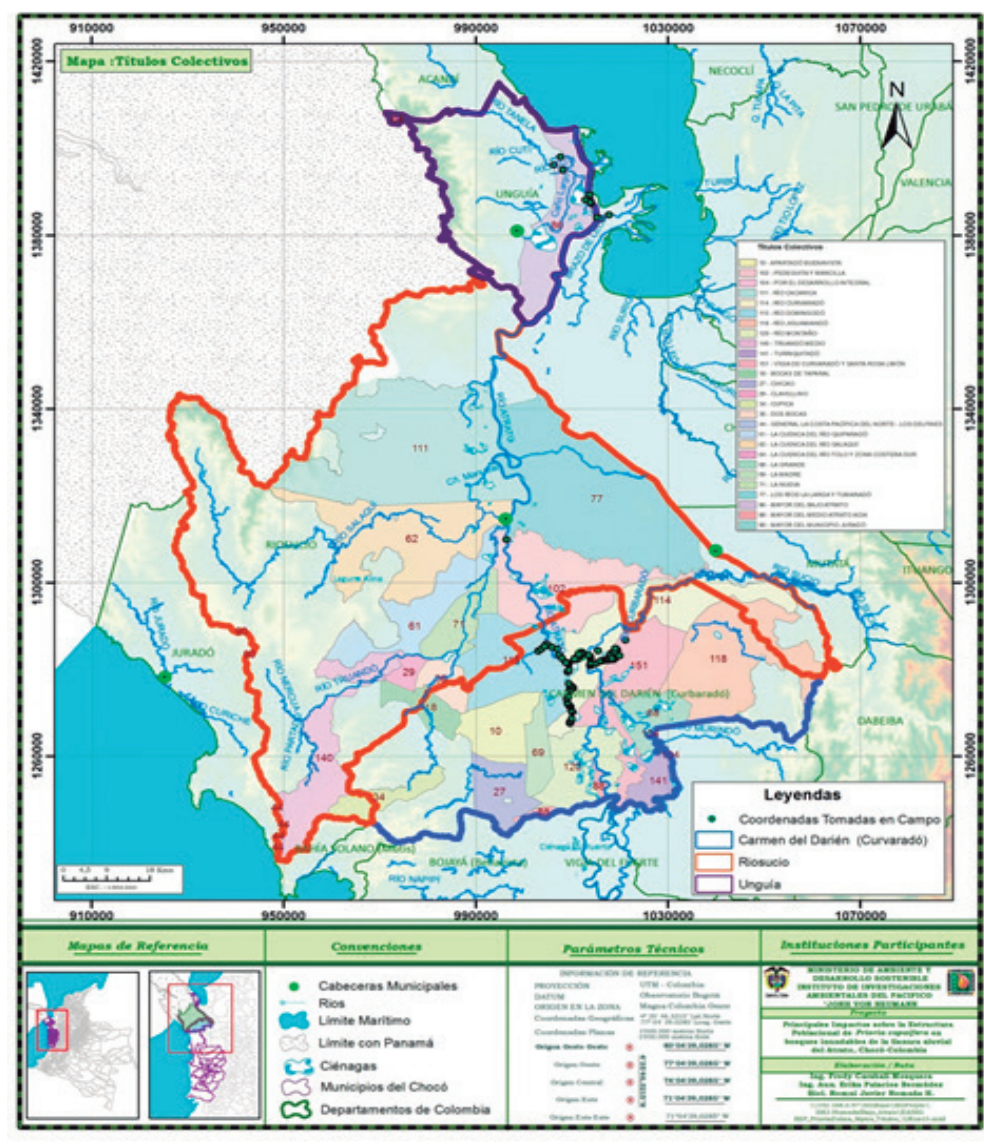

Figura 1. Área de estudio.

temas cenagosos y planos de inundación, alimentados por cauces de variado caudal en el área que a su vez conforman una serie de humedales en la zona de estudio, receptores de una variada y rica diversidad biológica. Los suelos de los planos inundables están sometidos a inundaciones periódicas, de magnitud y duración acorde con la posición fisiográfica, donde se forman complejos de humedales que posibilitan el desarrollo de comunidades vegetales diferenciables dominadas por pocas especies (Universidad Nacional de Colombia 1996, 1999).

El área presenta una serie de ambientes de gran importancia debido a los procesos hidrológicos y ecológicos que en ellos ocurren y la diversidad biológica que sustentan, constituyéndose en ambientes altamente productivos y hábitat crítico para especies seriamente amenazadas, que encuentran en los ecosistemas del Atrato condiciones que permiten suplir sus necesidades ecológicas y de esta manera contribuir al funcionamiento del ambiente, mediante procesos vitales (CODECHOCO y CORPOURABA 2006).
Métodos. El estado poblacional de $M$. arborescens en los sitios de muestro (Carmen de Darién, Riosucio y Unguía) se realizó en zonas aluviales y áreas influidas por humedales con diferentes niveles de intervención antrópica; en cada localidad realizaron recorridos aleatorios simples al azar a fin de seleccionar las potenciales áreas de muestreo; una vez seleccionadas se delimitaron cinco transectos de 20x50 mequivalentes a 0,5 ha, con distancias mínimas de $100 \mathrm{~m}$. Finalmente se censaron todos los individuos de la especie en dos estados de desarrollo plántulas y adultos (Gentry 1993; Phillips y Miller 2002), de igual forma, se registraron las especies asociadas con M. arborescens.

Las colecciones del material vegetal se realizaron teniendo en cuenta las normas específicas. A cada ejemplar colectado en campo se le tomaron los datos correspondientes a descripción morfológica, presencia de látex, exudados, resinas, olores, colores de órganos reproductivos, nombre vulgar y otras características vegetales importantes para la identificación.

Los ejemplares colectados fueron transportados al Herbario CHOCO de la Universidad Tecnológica del Chocó "Diego Luis Córdoba", Quibdó, Colombia, para su respectivo secado y posterior identificación, con la ayuda de claves taxonómicas (Gentry 1993) y las publicaciones especializadas en diferentes grupos botánicos (Forero y Gentry 1989, Rangel et al. 2004, Mahecha 2007) y diferentes bases de datos online (http:/www.biovirtual. unal.edu; http://www.biovirtual.unal. edu.co/ICN) entre otras, además de la 


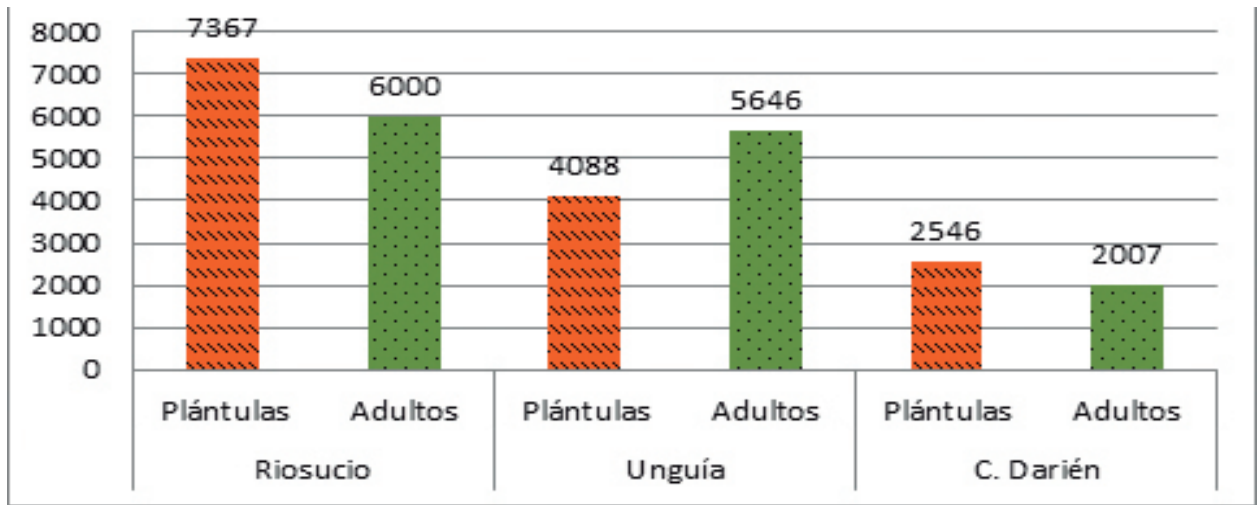

Figura 2. Estructura de la población de M. arborescens.

ayuda de taxónomos expertos. El análisis de los datos se realizó basado en la abundancia y frecuencia de aparición de individuos de M. arborescens; los cálculos se realizaron en Excel, utilizando el programa estadístico R versión 3.1.2.

Análisis de los datos. Para determinar si existen diferencias significativas entre la estructura de las poblaciones de $M$. arborescens en el Medio y Bajo Atrato, se aplicó la prueba de Kruskal-Wa1lis. Las diferencias de medias significativas se cuantificaron con intervalos de confianza (IC) al 95\%. El análisis de los datos se realizó con el paquete estadístico IBM SPSS Statistics ${ }^{\circledR} 19.0$ para Windows (IBM Corp., Armonk, NY).

\section{Resultados y discusión}

Estructura de la población de M. arborescens. Se registraron 27.654 individuos de $M$. arborescens en 1,5 ha distribuidos entre 14.001 plántulas y 13.653 adultos de una muestra de la población presente en tres localidades en la llanura aluvial del Atrato (Tabla 1); las plántulas fueron el estadío mejor representado (51\%), seguido de los adultos (49\%) para las 15 parcelas evaluadas. La localidad que mayor número de individuos presentó en los diferentes estadíos fue Riosucio con 13.367 individuos, seguido de Unguía con 9.739 individuos y la que menor número de individuos registro fue Carmen de Darién con 4.553.

La estructura poblacional de $M$. arborescens
Tabla 1. Densidad de la población de M. arborescens por localidad y estadío

\begin{tabular}{llll}
\hline Localidad & Estadío & $\begin{array}{c}\mathbf{N}^{\circ} \\
\text { individuos }\end{array}$ & $\begin{array}{c}\text { Densidad } \\
\text { poblacional }\end{array}$ \\
\hline C. Darién & Plántulas & 2.546 & 0,5092 \\
& Adultos & 2.007 & 0,4014 \\
Riosucio & Plántulas & 7.367 & 1,4734 \\
& Adultos & 6.000 & 1,2 \\
Unguía & Plántulas & 4.088 & 0,8176 \\
& Adultos & 5.646 & 1,1292 \\
Total & & 27.654 & \\
\hline
\end{tabular}

difiere entre los sitios muestreados, en Carmen de Darién y Riosucio; el estadío plántulas es superior al estadío adultos garantizando así la estabilidad de las poblaciones, mientras que en la localidad de Unguía, los individuos adultos superan en número a las plántulas (Figura 2). Lo anterior puede estar relacionado con las condiciones del lugar, el cual al permanecer inundado la mayor parte del año puede ser el sitio óptimo para el establecimiento de la especie, convirtiendo a esta población de la localidad en un bosque clímax, lo que a su vez puede estar limitando las estrategias de supervivencia de la especie en este estadío debido a la competencia por espacio.

Variaciones espaciales del número de individuos en diferentes estadíos de crecimiento. De acuerdo con los datos obtenidos, se encontró 


\section{Bioetnia Volumen 13, 2016}

una densidad de plántulas (plántulas $/ \mathrm{m}^{2}$ ) mayor en Riosucio (1,4-7.367 plántulas $/ \mathrm{m}^{2}$ ), intermedia en Unguía (1,1-4.088 plántulas $\left./ \mathrm{m}^{2}\right)$ y menor en Carmen de Darién (0,5-2.546 plántulas $\left./ \mathrm{m}^{2}\right)$.

Los resultados de este estudio corroboran lo manifestado por Gordon et al. (2000), quienes atribuyen que la densidad de individuos de esta especie varía de acuerdo con el sitio de establecimiento de la misma, por las diferencias en las características de cada lugar (microtopografía, composición y humedad del suelo y cambios en la profundidad del agua).

En este sentido la Figura 3 muestra la variabilidad de la densidad de $M$. arborescens de acuerdo con cada localidad. El Carmen de Darién presentó una densidad poblacional baja de 0,91 individuos $\mathrm{m}^{2}$, lo cual puede estar relacionado con microtopografía y los cambios en la profundidad del agua; en esta localidad las poblaciones de arracacho suelen encontrarse dispersas formando parches discontinuos, lo que concuerda con lo manifestado por Gordon et al. (2001), quienes determinaron que esta especie aumenta su densidad cerca de la orilla y decrece hacia áreas terrestres, situación que puede estar influyendo en el establecimiento de la especie en Carmen de Darién, donde las condiciones de suelo y topografía difieren de las otras localidades. Riosucio presentó la mayor densidad poblacional $\left(2,67\right.$ individuos $\left.\mathrm{m}^{2}\right)$; en esta localidad las poblaciones de esta especie se encuentran continuas formando densas poblaciones asociadas con la ribera del río Atrato, en las ciénagas conectadas a este y en los tributarios, lo que hace que las condiciones topográficas, la composición y humedad del sueloy la periodicidad del nivel del agua hayan creado las condiciones necesarias para el establecimiento de la especie.

En la localidad de Unguía los arracachales presentaron una densidad poblacional de 1,94 individuos $\mathrm{m}^{2}$, la cual es inferior comparada con la localidad de Riosucio; esto puede estar relacionado con su cercanía al Golfo de Urabá; en este sentido Colonnello y Medina (1998) manifiestan que las poblaciones de $M$. arborescens limitan su distribución por las concentraciones de salinidad, lo que podría haber influido en la baja densidad de la especie en esta localidad. Las cifras de densidad poblacional para cada uno de los estadíos y sitios muestreados difieren con lo obtenido por Gordon et al. (2000) en la laguna Grande del estado de Monagas, Venezuela, quienes reportan valores de hasta $7 \mathrm{ind} / \mathrm{m}^{2}$ para plántulas y hasta $7 \mathrm{ind} / \mathrm{m}^{2}$ para el estadío adulto. Lo anterior, puede estar relacionado con el tipo de ambiente; el citado en este estudio corresponde a un embalse cuyas condiciones ecológicas difieren con las del río Atrato en donde se desarrolló este estudio.

Un factor que puede estar influyendo la baja densidad de las poblaciones de arracacho en el bajo Atrato puede estar relacionado con los niveles

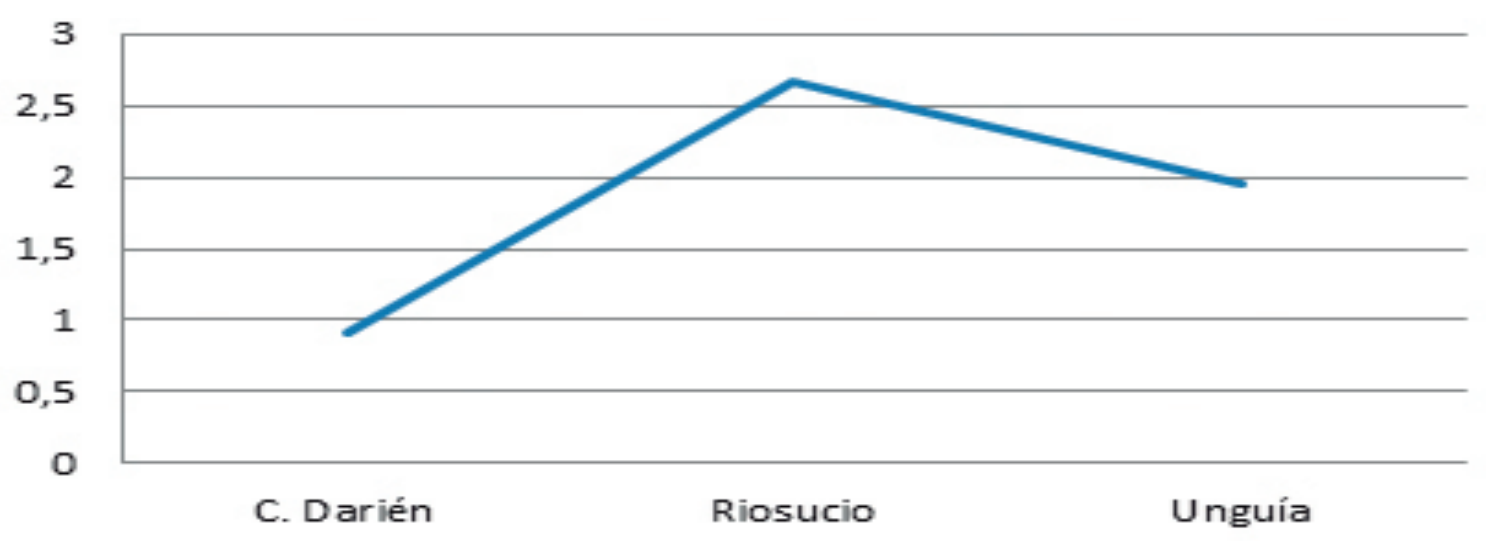

Figura 3. Densidad poblacional de $M$. arborescens en las localidades de Carmen de Darién, Riosucio y Unguía. 
de intervención ocasionados por diferentes actividades que se realizan en la zona, lo que induce al corte de la especie para la creación de rutas de navegabilidad.

M. arborescens tiene la capacidad de establecerse en áreas muy cercanas al mar, donde la influencia de este es baja, como en áreas alejadas sobre el delta de río Atrato; lo anterior se debe a su rango de distribución que va desde el nivel del mar, hasta los $200 \mathrm{msnm}$ en bancos arenosos hasta ríos de aguas negras, y en pantanos herbáceos donde se acumula la materia orgánica (Bunting 1995, Gordon 1996). En este sentido Gordon (2013) manifiesta que las características del sustrato no limitan el establecimiento y supervivencia de la especie, sino la presencia de agua y su nivel de inundación.

La estructura poblacional de $M$. arborescens en general presentó patrones de buen estado de conservación, en dos de las localidades muestreadas; el número de individuos en estadío plántulas superaron al estadío adulto, garantizando así el mantenimiento de la especie en el tiempo y el espacio; las estrategias reproductivas y de adaptación y supervivencia de esta especie la facultan para colonizar los ambientes fluviolacustres donde se establece y desarrolla.

Los arracachales de la llanura aluvial del río Atrato prestan una serie de bienes y servicios, se constituye el hábitat de numerosas especies sobre todo aves, sirven de alimento a peces y otros animales acuáticos, quienes encuentran en esta asociación las condiciones necesarias para su supervivencia; forman un sitio de pesca para los pobladores locales. De igual forma estas asociaciones, se constituyen en fuente de energía y materiales para algunos niveles tróficos del ecosistema.

\section{Agradecimientos}

Alos consejos comunitarios locales de Carmen de Darién, Riosucio y Unguía por permitirnos rea- lizar este tipo de investigaciones en su territorio, por el acompañamiento y la trasmisión de su conocimiento relacionada con los cambios ocurridos a este tipo de ambientes a través del tiempo y por facilitarnos la logística necesaria para la movilidad en los puntos de cada muestreo. Alos compañeros Yuber Palacios, Yair Cuesta Nagles, Eric Yair Cuesta Ríos y a cada uno de los participantes del proyecto "Espacialización de sitios por criterios utilizado para su incorporación a la estructura ecológica principal del Chocó Biogeográfico", por la toma y facilitación de los datos.

\section{Literatura citada}

Aldana J. 2011. Transformación físico-espacial de los paisajes boscosos del sector sur del Parque Nacional Natural los Katíos (1989-2010). Perspect Geogr. 16: 31-54. URL disponible en: http://revistas.uptc.edu.co/ revistas/index.php/perspectiva/article/view/1749/1744

Bunting GS. 1995. Araceae(Montrichardia).In: Steyermark, J, Berry P, Molst B, Yatskievych K (eds.). Flora of the Venezuelan Guayana. Vol. 2.Pteridophytes, Spermatophytes, Acanthaceae-Araceae. Chicago: The University of Chicago Press Books; 706 pp.

Cook CDK. 1996. Aquatic plant book. $2^{\text {nd }}$ ed. Amsterdam: SPB Academic Pub.; 228 pp.

Colonnello G, Medina E. 1998. Vegetation changes induced by dam construction in tropical estuary: the case of the Mánamo river, Orinoco Delta (Venezuela). Plant Ecol. 139 (2): 145-54. URL disponible en: https://link. springer.com/article/10.1023/A\%3A1009785118019

Corporación Autónoma Regional para el Desarrollo Sostenible del Chocó (CODECHOCÓ) y Corporación para el Desarrollo Sostenible de Urabá (CORPOURABÁ). 2006. Plan de manejo integrado de los humedales del medio y Bajo Atrato. Quibdó: CODECHOCÓ, CORPOURABÁ.

Forero E, Gentry A. 1989. Lista anotada de plantas del departamento del Chocó, $N^{o}$ 10. Bogotá: Editorial Guadalupe Ltda.

Gentry AH. 1993. Riqueza de especies del Pacífico. In: Leyva P(ed.). Colombia Pacífico I. Bogotá:Fondo FEN.

Gordon E, Suárez-Villasmil LM, Polanco L, Barreto-Pittol EM. 2013. Distribución geográfica y variación foliar de Montrichardia arborescens en Venezuela. Acta Biol Venez. 33 (1-2): 23-42. URL disponible en: http://www.academia.edu/18377210/DISTRIBUCI\%C3\%93N_GEOGR\%C3\%81FICA_Y VARIA- 


\section{Bioetnia Volumen 13, 2016}

CI\%C3\%93N FOLIAR DE Montrichardia arborescens EN VENEZUELA

Gordon E, Pardo MJ. 2012. Riqueza y composición de especies promisorias de un sector de los Llanos Orientales, Venezuela. Acta Biol Venez. 32 (2): 99-180. URL disponible en: http://saber.ucv.ve/ojs/index.php/ revista abv/article/view/6358

Gordon E, Polanco L, Peña C. 2001. Contribución a la ecología de Montrichardia arborescens (L.) Schott (Araceae). III. Distribución a lo largo del gradiente de profundidad del agua. Acta Biol Venez. 21 (2): 39-47. URL disponible en: http://saber.ucv.ve/ojs/index.php/ revista abv/article/view/4114

Gordon E, Polanco L, Peña C. 2000. Contribución a la ecología de Montrichardia arborescens (L.) Schott (Araceae). I. Demografía. Acta Biol Venez. 20 (4): 51 64. URL disponible en: http://saber.ucv.ve/ojs/index. $\mathrm{php} / \mathrm{revista}$ abv/article/view/4136/3957

Gordon, E. 1996. Caracterización de la vegetación acuática vascular y de los bancos de semillas en Laguna Grande, estado Monagas. Tesis Doctoral, Facultad de Ciencias, Universidad Central de Venezuela; 291 pp.

Mahecha VG. 1997. Fundamentos y metodología para la identificación de plantas. Proyecto Biopacífico. Bogotá: Ministerio del Media Ambiente-Gfi'I- PNUD; pp. 59-145.

Peña C, Gordon E, Ramírez A. 2000. Descomposición de Montrichardia arborescens (L) Schott (Araceae). En: Péfaur JE (ed.). Ecología Latinoamericana. Actas III Congreso Latinoamericano de Ecología, Mérida; 153-161 pp.

Phillips OL, Miller JS. 2002. Global patterns of plant diversity: Alwyn H. Gentry`s forest transect data set. Monographs in Systematic Botany from the Missouri Botanical Gatden. 89: 1-37.

Prieto-CA, Rudas-Ll A, Rangel-Ch JO, Gónima-G L, Serrano H. 2004. La vegetación del Darién colombiano: una aproximación histórica aplicada a la interpretación satelital y videográfica. En: Rangel-Ch JO (ed.). Colombia Diversidad Biótica IV. El Chocó Biogeográfico/ Costa Pacífica. Bogotá: Universidad Nacional de Colombia, Instituto de Ciencias Naturales, Conservación
Internacional; 997 pp. URL disponible en: https://issuu. com/diversidadbiotica/docs/name120ff4

Rangel-Ch JO. 2004a. Amenazas a la biota y a los ecosistemas del Chocó Biogeográfico. En: Rangel-Ch JO (ed.). 2004. Colombia Diversidad Biótica IV. El Chocó Biogeográfico/Costa Pacífica. Bogotá: Universidad Nacional de Colombia, Instituto de Ciencias Naturales, Conservación Internacional; 997 pp. URL disponible en: https://issuu.com/diversidadbiotica/docs/dbivcap19.-amenazas-a-la-biota-y-los-ecosistemas

Rangel Ch. J.O. 2004b. La vegetación del Chocó Biogeográfico de Colombia. En: Rangel-Ch JO (ed.). 2004. Colombia Diversidad Biótica IV. El Chocó Biogeográfico/Costa Pacífica. Bogotá: Universidad Nacional de Colombia, Instituto de Ciencias Naturales, Conservación Internacional; 997 pp. URL disponible en: https:// issuu.com/diversidadbiotica/docs/nameb2c524

Rangel-Ch JO, Rivera-Díaz O, Giraldo-Cañas D, Parra-O C, Murillo JC, Gil I, et al. 2004. Catálogo de espermatófitos en el Chocó Biogeográfico. En: Rangel-Ch JO (ed.). 2004. Colombia Diversidad Biótica IV. El Chocó Biogeográfico/Costa Pacífica. Bogotá: Universidad Nacional de Colombia, Instituto de Ciencias Naturales, Conservación Internacional; 997 pp. URL disponible en: https://issuu.com/diversidadbiotica/ docs/name115624/86

Universidad Nacional de Colombia. 1996. Diagnóstico de las existencias de la palma murrapo o naidi (Euterpe oleracea M.) y del componente arbóreo en un área del municipio de Vigía del Fuerte, región del Medio Atrato, Colombia. Medellín: Universidad Nacional de Colombia.

Universidad Nacional de Colombia. 1999. Observaciones preliminares de la demografía y el crecimiento de Euterpe oleracea en la región del Medio Atrato. Medellín: Universidad Nacional de Colombia.

Valoyes CZ, Ramírez MG, Klinger W, Carabalí MF. 2012. Estructura ecológica principal del Chocó Biogeográfico, según criterio de diversidad y singularidad de especies y ecosistemas. Bioetnia 9 (2): 115-35. URL disponible en: http://iiap.org.co/documents/6c71786ff4630ab11074df6bb42f7bd5.pdf 